\title{
Black Hole Remnants at the LHC
}

\author{
L. Bellagamba ${ }^{b *}$ R. Casadio ${ }^{a, b \dagger}, \quad$ R. Di Sipio ${ }^{a, b \ddagger}$ and V. Viventi ${ }^{a \S}$ \\ ${ }^{a}$ Dipartimento di Fisica, Università di Bologna \\ via Irnerio 46, 40126 Bologna, Italy \\ ${ }^{b}$ Istituto Nazionale di Fisica Nucleare, Sezione di Bologna \\ via Irnerio 46, 40126 Bologna, Italy
}

October 23, 2018

\begin{abstract}
We investigate possible signatures of black hole events at the LHC in the hypothesis that such objects will not evaporate completely, but leave a stable remnant. For the purpose of defining a reference scenario, we have employed the publicly available Monte Carlo generator CHARYBDIS2, in which the remnant's behavior is mostly determined by kinematic constraints and conservation of some quantum numbers, such as the baryon number. Our findings show that electrically neutral remnants are highly favored and a significantly larger amount of missing transverse momentum is to be expected with respect to the case of complete decay.
\end{abstract}

\section{Introduction}

Models with large extra dimensions [1, 2] allow for the fundamental scale of gravity $M_{\mathrm{G}}$ to be as low as the electro-weak scale $\left(M_{\mathrm{G}} \simeq 1 \mathrm{TeV}\right)$, and microscopic black holes (BH) may therefore be produced in our accelerators (for reviews, see, e.g., Refs. 3]). Once a BH is formed, and the balding phase is over, the Hawking radiation [4] sets off. The standard description of this effect is based on the canonical Planckian distribution for the emitted particles, which implies the life-time of microscopic BHs is very short, of the order of $10^{-26} \mathrm{~s}[5]$. This picture (restricted to the ADD scenario [1]) has been implemented in several numerical codes [6, 7, 8, 9, 10, 11, 12, which have been mainly designed to extract information that will allow us to identify $\mathrm{BH}$ events at the Large Hadron Collider (LHC) (see Ref. [13] for a search of BH signatures with the first data collected by the LHC and Ref. [14] for some theoretical criticisms on the validity of that analysis).

It is important to recall that the end-stage of the $\mathrm{BH}$ evaporation remains an open issue (see, e.g., Refs. [15, 16, 17]), because we do not yet have a confirmed theory of quantum gravity. In fact, the semiclassical Hawking temperature grows without bound, as the BH mass decreases, which can

*lorenzo.bellagamba@bo.infn.it

†roberto.casadio@bo.infn.it

${ }^{\ddagger}$ riccardo.disipio@bo.infn.it

§vi.strawberry@gmail.com 
be viewed as a sign of the lack of predictability of perturbative approaches ${ }^{1}$. This is an important issue also on a purely experimental side, since deviations from the Hawking law for small BH mass (near the fundamental scale $M_{\mathrm{G}}$ ) could actually lead to detectable signatures.

Our ignorance of the late stages of the $\mathrm{BH}$ evaporation is usually bypassed in the numerical codes by letting the microscopic BH decay into a few standard model particles as an (arbitrary) lower mass is reached, but the possibility of ending the evaporation by leaving a stable remnant has also been considered [20, 21, 22]. The purpose of this report is precisely to study the case in which BHs stop emitting and leave a long-lived (or even stable) remnant of mass $M \simeq M_{\mathrm{G}}$.

For this purpose, we have employed the Monte Carlo code CHARYBDIS2 [12], which is presently the only generator which natively supports remnant BHs, as we shall describe in Section 3 . In Section 4, we report results from test runs and describe in detail both the primary emission and final output obtained using Pythia [23. We shall use units with $c=\hbar=1$ and the Boltzmann constant $k_{\mathrm{B}}=1$.

\section{Black hole production and evolution}

In the ADD scenario [1, a microscopic $\mathrm{BH}$ with horizon radius shorter than the typical size of the extra dimensions is usually approximated by a $(4+d)$-dimensional solution of the vacuum Einstein equations. In the simplest case of a non-rotating $\mathrm{BH}$, this leads to the following expression for the horizon radius,

$$
R_{\mathrm{H}}=\frac{1}{\sqrt{\pi} M_{\mathrm{G}}}\left(\frac{M}{M_{\mathrm{G}}}\right)^{\frac{1}{d+1}}\left(\frac{8 \Gamma\left(\frac{d+3}{2}\right)}{d+2}\right)^{\frac{1}{d+1}}
$$

where $M$ is the $\mathrm{BH}$ mass, $\Gamma$ the Gamma function, and the temperature associated with the horizon is given by

$$
T_{\mathrm{H}}=\frac{d+1}{4 \pi R_{\mathrm{H}}} .
$$

At the LHC, a BH could form by colliding two protons. The total $\mathrm{BH}$ cross section can be estimated from the geometrical hoop conjecture [24] as

$$
\sigma(M) \approx \pi R_{\mathrm{H}}
$$

In order to determine the total production cross section, this expression must be rescaled according to the parton luminosity approach as

$$
\left.\frac{d \sigma}{d M}\right|_{p p \rightarrow B H+X}=\frac{d L}{d M} \hat{\sigma}\left(a b \rightarrow B H ; \hat{s}=M^{2}\right),
$$

where $a$ and $b$ represent the partons which form the $\mathrm{BH}, \sqrt{\hat{s}}$ is their centre-mass energy and

$$
\frac{d L}{d M}=\frac{2 M}{s} \sum_{a, b} \int_{M^{2} / s}^{1} \frac{d x_{a}}{x_{a}} f_{a}\left(x_{a}\right) f_{b}\left(\frac{M^{2}}{s x_{a}}\right),
$$

\footnotetext{
${ }^{1}$ For reviews of the more consistent microcanonical description of $\mathrm{BH}$ evaporation in which energy conservation is granted, see Refs. [18, 19.
} 
where $f_{i}\left(x_{i}\right)$ are parton distribution functions (PDF) and $\sqrt{s}$ the LHC centre-mass collision energy (up to $7 \mathrm{TeV}$ presently, with a planned maximum of $14 \mathrm{TeV}$ ). Of course, semiclassical BHs will only form above a minimum mass (presumably) larger than $M_{\mathrm{G}}$ [14, 25].

Once formed, the $\mathrm{BH}$ begins to evolve. In the standard picture, the decay process can be divided into three characteristic stages:

Balding phase: the $\mathrm{BH}$ radiates away the multipole moments inherited from the initial configuration and reaches a hairless state. A fraction of the initial mass will also be radiated as gravitational radiation.

Evaporation phase: the $\mathrm{BH}$ loses mass via the Hawking effect. It first spins down by emitting the initial angular momentum, after which it proceeds with the emission of thermally distributed quanta. The radiation spectrum contains all the Standard Model (SM) particles, (emitted on our brane), as well as gravitons (also emitted into the extra dimensions). For this tags, it is crucial to have a good estimate of the grey-body factors [26, 27].

Planck phase: the $\mathrm{BH}$ has reached a mass close to the effective Planck scale $M_{\mathrm{G}}$ and falls into the regime of quantum gravity. It is generally assumed that the $\mathrm{BH}$ will either completely decay into SM particles [5] or a (meta-)stable remnant is left, which carries away the remaining energy [20].

We will here focus on the possibility that the third phase ends by leaving a stable remnant.

\section{Remnants and a Monte Carlo code}

Several Monte Carlo codes which simulate the production and decay of micro-BHs are now available (see, e.g. Refs. [6, 7, 8, 9, 10, 11, 12]). The only code which presently implements the description of a remnant is CHARYBDIS2 [12] 2. Such a description is mostly based on kinematic and quantum number constraints, so that the remnant will necessarily have zero baryon number. The production and evaporation phases are better modeled, from a theoretical point of view, although they could still be improved. For example, the production phase is described using bounds from trapped surface formation, which might lead to overestimate the amount of missing energy in gravitational radiation [28]. Further refinements have also been investigated for the Hawking spectra [29, 10].

A list of the relevant adjustable parameters in the code and the values we used in the simulations, is given in Table 1. In the study we present here, we simulated the different scenarios in $p p$ collisions with total centre-mass energy of both $\sqrt{s}=7 \mathrm{TeV}{ }^{3}$ and $14 \mathrm{TeV}$.

It is particularly important to describe in details the role of one of the kinematic constraints, namely KINCUT. When set to TRUE, the generator will not allow the emission of Hawking quanta that reduce the $\mathrm{BH}$ mass $M$ below $M_{\mathrm{G}}$, whereas KINCUT=FALSE will allow the last decay particle to have an energy as large as the BH's (but no more). Let us then see what will likely happen for the last emission, when the $\mathrm{BH}$ has already evaporated down to a mass $M_{\mathrm{LE}} \gtrsim M_{\mathrm{G}}$. The generator will try to simulate an Hawking particle with energy $\omega$ determined by the Planckian distribution with a temperature $T_{\mathrm{H}}=T_{\mathrm{H}}\left(M_{\mathrm{LE}}\right)$ and small enough to ensure energy conservation. Roughly speaking (that is, neglecting the BH kinetic energy), this means $T_{\mathrm{H}}\left(M_{\mathrm{LE}}\right) \simeq \omega<M_{\mathrm{LE}}$.

\footnotetext{
${ }^{2}$ This code has been used to study the remnants of non-commutative BHs in Ref. 22

${ }^{3}$ This case was mostly included for a comparison with the CMS analysis in Ref. 13, although there are arguments which show that $7 \mathrm{TeV}$ might not be theoretically enough to produce semiclassical BHs [14].
} 


\begin{tabular}{|c|c|c|}
\hline Parameter & Description & Used values \\
\hline$M_{\mathrm{G}}$ & Fundamental gravitational scale & $2-4 \mathrm{TeV}$ \\
\hline $\mathrm{D}$ & Total number of dimensions & 6,8 \\
\hline MINMSS & minimum initial BH mass & $2 \times M_{\mathrm{G}}$ \\
\hline MAXMSS & maximum initial BH mass & $\sqrt{s}$ \\
\hline RMSTAB & main switch for stable BH remnant & TRUE/FALSE \\
\hline KINCUT & kinematic condition for BH remnant & TRUE/FALSE \\
\hline
\end{tabular}

Table 1: Most relevant parameters of CHARYBDIS2 and their values for the different simulated samples used in this study.

Since the temperature 2.2 increases for decreasing $M$, it is likely the attempted last $\omega$ will carry away enough energy to reduce $M$ below $M_{\mathrm{G}}$. If KINCUT=TRUE, this process is forbidden and the generator simply stops and leaves a remnant of mass $M_{\mathrm{LE}}>M_{\mathrm{G}}$. If KINCUT=FALSE, the process is allowed and the remnant mass will be $M \simeq M_{\mathrm{LE}}-\omega<M_{\mathrm{G}}$. The effect of KINCUT is clearly visible in Fig. 1, which shows the remnant mass distributions for samples generated at $\sqrt{s}=7 \mathrm{TeV}$ with $M_{\mathrm{G}}=2 \mathrm{TeV}$, in $D=6$ and 8 . If KINCUT=FALSE the last decay step, which allows the remnant to access masses lower than $M_{\mathrm{G}}$, typically releases a lot of energy, producing low mass remnants. On the other hand KINCUT=TRUE forbids remnant masses below $M_{\mathrm{G}}$, thus producing an increase of events as the remnant mass approaches $M_{\mathrm{G}}$ and long tails towards higher values. These scenarios are in agreement with the results we shall show in the next Section.

From a theoretical point of view, one expects a remnant mass around $M_{\mathrm{G}}$ (see, e.g., Ref. [25]). The above scenarios are therefore, in a sense, complementary, with the KINCUT=FALSE case perhaps less sound.

\section{Simulations}

The standard setting for CHARYBDIS2 simulates the evaporation of a microscopic BH according to Hawking's law, until the black hole mass $M$ reaches the minimum value, which is assumed equal to the fundamental scale of gravity $M_{\mathrm{G}}$. The $\mathrm{BH}$ subsequently decays into a few bodies $\left(N_{\mathrm{f}}=2, \ldots, 6\right)$ simply according to phase space. As described in the previous Section, we are instead interested in the study of scenarios with a residual stable BH remnant in the final state.

In order to compare the output of the different scenarios (no stable remnant, stable remnant with KINCUT=TRUE/FALSE) we have generated Monte Carlo samples varying the parameters listed in Table 1 and assuming two different centre mass energy for the LHC, namely 7 and $14 \mathrm{TeV}$. As pointed out in Table 1, the mass of the produced $\mathrm{BH}$ has been required to be at least $2 M_{\mathrm{G}}$ in order to avoid the region where the classical theory, used to evaluate the production cross section, is no longer valid (but see also Ref. [14]). The other parameters have been fixed to the CHARYBDIS2 default values. Table 2 summarizes the characteristics of the generated samples, including the $t \bar{t}$ production process which is one of the most relevant backgrounds. The table also contains the event yields normalized to an integrated luminosity of $1 \mathrm{fb}^{-1}$ for few basic selection requirements applied at the hadronization level, as described in details in Section 5 . 


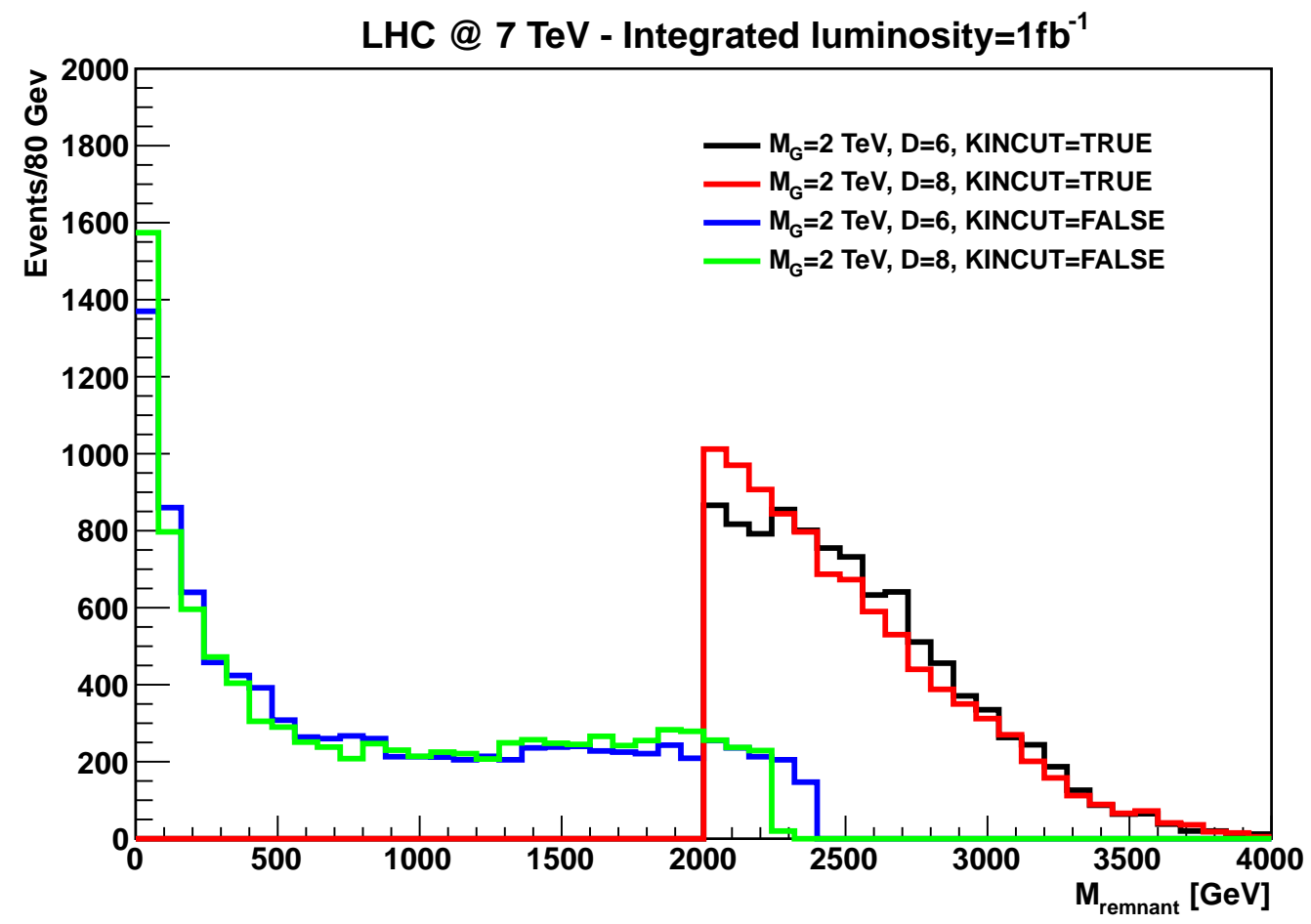

Figure 1: Remnant mass for different values of the KINCUT parameters.

\subsection{Primary Emission}

Let us first examine the "primary" $\mathrm{BH}$ emission, namely the particles produced by direct $\mathrm{BH}$ evaporation, before parton evolution and hadronization are taken into account. This emission is the direct output of CHARYBDIS2. Fig. 2 shows the produced final state particles and the particle multiplicity for $\sqrt{s}=7 \mathrm{TeV}, M_{\mathrm{G}}=2 \mathrm{TeV}$, in $D=8$ and for the different remnant conditions. The final state particles, listed in Fig. 2, panels a), c) and e), are grouped in SM particles (the first three blocks contain quarks, leptons and bosons, respectively), gravitons $(\mathrm{G})$, neutral $\left(\mathrm{R}^{0}\right)$ and charged $\left(\mathrm{R}^{ \pm}\right) \mathrm{BH}$ remnants. The remnants are neutral in the large majority of the cases, with only a few percent of the events ending in a charged remnant. The neutral remnants behave as weakly interacting massive particles (WIMPs), producing a striking signal of missing transverse energy and a topology quite different from the usual case of total $\mathrm{BH}$ evaporation.

\subsection{Final Output}

The primary output includes unstable particles, along with quarks and gluons that have to be processed by a parton shower Monte Carlo in order to produce the final state observables (hadron level). We used PYTHIA [23] to model the parton evolution and hadronization of quarks and gluons emitted during the $\mathrm{BH}$ evaporation, as well as to treat the unstable particle decays and the proton remnants. This produced the final features of the events as would be seen by the LHC detectors. Besides the signal BH samples, also one of the most relevant SM backgrounds for such topologies, the process $p p \rightarrow t \bar{t} X$, has been generated using the NLO generator POWHEG [30], 


\begin{tabular}{|c|c|c|c|c|c|c|c|}
\hline \multicolumn{8}{|c|}{$p p$ collision $\sqrt{s}=7 \mathrm{TeV}$} \\
\hline Process & $M_{\mathrm{G}}[\mathrm{TeV}]$ & $\mathrm{D}$ & $\sigma[\mathrm{pb}]$ & remnant & $P_{T}^{\text {lep }}>0.5 \mathrm{TeV}$ & $P_{T}^{\text {miss }}>0.5 \mathrm{TeV}$ & $E_{T}>2 \mathrm{TeV}$ \\
\hline $\mathrm{BH}$ & 2.0 & 6 & 0.017 & KINCUT $=$ TRUE & $0.18 \pm 0.02$ & $11.8 \pm 0.1$ & $0.39 \pm 0.03$ \\
\hline $\mathrm{BH}$ & 2.0 & 6 & $"$ & KINCUT $=$ FALSE & $0.51 \pm 0.03$ & $14.9 \pm 0.2$ & $7.1 \pm 0.1$ \\
\hline $\mathrm{BH}$ & 2.0 & 6 & $"$ & no remnant & $0.94 \pm 0.04$ & $1.74 \pm 0.05$ & $15.8 \pm 0.2$ \\
\hline $\mathrm{BH}$ & 2.0 & 8 & 0.052 & KINCUT $=$ TRUE & $0.49 \pm 0.05$ & $33.9 \pm 0.4$ & $1.69 \pm 0.09$ \\
\hline $\mathrm{BH}$ & 2.0 & 8 & $"$ & KINCUT $=$ FALSE & $1.57 \pm 0.09$ & $43.0 \pm 0.5$ & $22.9 \pm 0.3$ \\
\hline $\mathrm{BH}$ & 2.0 & 8 & $"$ & no remnant & $3.2 \pm 0.1$ & $5.4 \pm 0.2$ & $46.5 \pm 0.5$ \\
\hline $\mathrm{BH}$ & 2.5 & 8 & $1.74 \times 10^{-2}$ & KINCUT $=$ TRUE & $0.0023 \pm 0.0002$ & $0.130 \pm 0.001$ & $0.0169 \pm 0.0005$ \\
\hline $\mathrm{BH}$ & 2.5 & 8 & $"$ & KINCUT $=$ FALSE & $0.0070 \pm 0.0003$ & $0.151 \pm 0.002$ & $0.112 \pm 0.001$ \\
\hline $\mathrm{BH}$ & 2.5 & 8 & $"$ & no remnant & $0.0119 \pm 0.0005$ & $0.0225 \pm 0.0006$ & $0.162 \pm 0.002$ \\
\hline$t \bar{t}$ & - & - & 84 & - & 0 & $2.0 \pm 1.2$ & $5.4 \pm 1.9$ \\
\hline \multicolumn{8}{|c|}{$p p$ collision $\sqrt{s}=14 \mathrm{TeV}$} \\
\hline Process & $M_{\mathrm{G}}[\mathrm{TeV}]$ & $\mathrm{D}$ & $\sigma[\mathrm{pb}]$ & remnant & $P_{T}^{\text {lep }}>1 \mathrm{TeV}$ & $P_{T}^{\text {miss }}>1 \mathrm{TeV}$ & $E_{T}>4 \mathrm{TeV}$ \\
\hline $\mathrm{BH}$ & 3.5 & 6 & 0.040 & KINCUT $=$ TRUE & $0.30 \pm 0.03$ & $24.8 \pm 0.3$ & $0.47 \pm 0.04$ \\
\hline $\mathrm{BH}$ & 3.5 & 6 & $"$ & KINCUT $=$ FALSE & $1.01 \pm 0.06$ & $32.7 \pm 0.4$ & $10.4 \pm 0.2$ \\
\hline $\mathrm{BH}$ & 3.5 & 6 & $"$ & no remnant & $1.82 \pm 0.08$ & $3.67 \pm 0.12$ & $33.5 \pm 0.4$ \\
\hline $\mathrm{BH}$ & 4.0 & 6 & $3.6 \times 10^{-3}$ & KINCUT=TRUE & $0.037 \pm 0.004$ & $2.45 \pm 0.03$ & $0.089 \pm 0.006$ \\
\hline $\mathrm{BH}$ & 4.0 & 6 & $"$ & KINCUT $=$ FALSE & $0.102 \pm 0.006$ & $3.02 \pm 0.03$ & $1.44 \pm 0.02$ \\
\hline $\mathrm{BH}$ & 4.0 & 6 & $"$ & no remnant & $0.198 \pm 0.008$ & $0.36 \pm 0.01$ & $3.18 \pm 0.03$ \\
\hline$t \bar{t}$ & - & - & 469 & - & 0 & 0 & $0.75 \pm 0.75$ \\
\hline
\end{tabular}

Table 2: List of generated Monte Carlo samples. Also reported are the expected numbers of events produced at the LHC for an integrated luminosity of $1 \mathrm{fb}^{-1}$ at $\sqrt{s}=7 \mathrm{TeV}$ and $14 \mathrm{TeV}$ after basic selection cuts on the $P_{T}$ of the leading lepton (electron or muon), missing $P_{T}$ and visible transverse energy, for different values of $M_{\mathrm{G}}$ and $D$. The expected events for one of the most relevant SM process, the $t \bar{t}$ production, are also reported.

interfaced with PYTHIA for the parton shower, hadronization and particle decays. At least one of the two top quarks was requested to decay in either $e, \mu$ or $\tau$.

\section{Results and outlook}

In order to point out the main characteristics of the different scenarios under study and the possibility to observe a signal over the SM background, we have applied a basic selection to the hadron level Monte Carlo samples using the following event variables:

- $P_{T}^{\text {lep }}$, defined as the transverse momentum of the leading lepton $(e$ or $\mu)$ with $\left|\eta^{\text {lep }}\right|<2.5$, where $\eta^{\text {lep }}$ is the lepton pseudorapidity;

- the missing transverse momentum,

$$
P_{T}^{\text {miss }}=\sqrt{\left(\sum_{i} P_{x_{i}}\right)^{2}+\left(\sum_{i} P_{y_{i}}\right)^{2}},
$$

where $P_{x_{i}}$ and $P_{y_{i}}$ are the cartesian $x$ and $y$ components of the momentum of the $i^{\text {th }}$ particle, and $i$ runs over all the undetectable final state particles: neutrinos, gravitons and neutral $\mathrm{BH}$ remnant;

- the visible transverse energy,

$$
E_{T}^{\mathrm{vis}}=\sum_{k}\left(\sqrt{P_{x_{k}}^{2}+P_{y_{k}}^{2}}\right)
$$


where $k$ runs over all the detectable final state particles.

The choice of the above simple variables is motivated by the fact that the observation of final state particles with transverse momentum of several hundreds of GeV's is the typical signal for the decay of a large mass state. In particular, the requirement of high-energy leptons and/or high missing transverse momentum allows to cope with the huge QCD background at the LHC. Since BHs decay democratically to all SM particles, the search for extremely energetic leptons is, in this context, one of the most direct way to look for deviations from the SM predictions.

However, in case of stable neutral remnants, the lepton signal can be depressed by the fact that a relevant fraction of the $\mathrm{BH}$ mass is not available in the decay. On the other hand, the neutral remnant, behaving as a WIMP, will carry away a lot of energy and enhance the missing transverse energy signal. This is clearly visible in Figs. 3 and 4 , where we show, for two representative cases at $\sqrt{s}=7$ and $14 \mathrm{TeV}$, the $P_{T}$ of the leading lepton, the $P_{T}^{\text {miss }}$ and the $E_{T}^{\mathrm{vis}}$ for the different scenarios. The $t \bar{t}$ background is also displayed.

The main characteristics of the different cases are further clarified in Fig. 5, which shows, for a representative case at $\sqrt{s}=7 \mathrm{TeV}$, the event distribution in the $E_{T}^{\mathrm{vis}}-P_{T}^{\text {miss }}$ plane for the different remnant scenarios and for the $t \bar{t}$ background. If the BH decays completely, the events are concentrated at relatively low $P_{T}^{\text {miss }}$ and very high $E_{T}^{\mathrm{vis}}$, whereas, in the case of a stable remnant, a shift towards much higher values of $P_{T}^{\text {miss }}$ and consequently lower $E_{T}^{\mathrm{vis}}$ is clearly visible. As already stressed in Section 3, the control parameter KINCUT sets two extreme and probably not realistic descriptions of the stable remnant. Nevertheless, given the large uncertainties in the theoretical picture, these two borderline scenarios allow to quantify the possible spread of the relevant experimental observables.

Table 2 summarizes the expected event yields, after basic selection cuts on the event variables described above, for the different BH scenarios and for the $t \bar{t}$ background 4 . The reported values are normalized to an integrated luminosity of $1 \mathrm{fb}^{-1}$ for $\sqrt{s}=7$ and $14 \mathrm{TeV}$. The results show that stable remnant scenarios up to $M_{\mathrm{G}} \lesssim 2 \mathrm{TeV}$ can be already probed with the $5 \mathrm{fb}^{-1}$ of integrated luminosity collected by the LHC during the last year of data taking. During the year 2012, we expect 4-to-5 times more integrated luminosity, which will allow us to probe the fundamental mass in the range $2 \mathrm{TeV} \lesssim M_{\mathrm{G}} \lesssim 2.5 \mathrm{TeV}$. The sensitivity to higher values could be gained only by increasing the LHC energy, since the production cross section for larger mass state is limited by the parton density functions of the proton, which steeply decrease going to larger momentum fraction of the partons. As shown in the second part of Table 2, $M_{\mathrm{G}} \simeq 4 \mathrm{TeV}$ could then be probed in the future, when the LHC runs at $\sqrt{s}=14 \mathrm{TeV}$.

Our findings should be mainly considered as an attempt to outline a reference picture, which can stimulate further investigations in such interesting stable BH remnant scenarios. Besides including specific theoretical models for BH remnants, further developments should consider other possible SM backgrounds (single-top, boson and di-boson production) which, even if less important than $t \bar{t}$, can give non-negligible contributions. The QCD production of events with two or more jets should also be considered due to its huge cross section. Since such processes can contribute to the background only for detector effects leading to bad reconstruction of the experimental observables, a reliable estimation of the expected background cannot disregard a detailed detector simulation.

\footnotetext{
${ }^{4}$ In the $t \bar{t}$ process, the high energy tails of the selection variables are not well populated due to the exponential decrease of the production rate with the energy. Anyway, despite possible statistical fluctuations, the contribution of the background in the signal region is very limited, given the very different high energy behaviour, as is clearly shown in Figs. 3 and 4 .
} 


\section{Acknowledgements}

We would like to thank the developers of CHARYBDIS2, in particular M.O.P. Sampaio and B.R. Webber, for very useful suggestions and comments.

\section{References}

[1] N. Arkani-Hamed, S. Dimopoulos and G. Dvali, Phys. Lett. B 429, 263 (1998); Phys. Rev. D 59, 0806004 (1999); I. Antoniadis, N. Arkani-Hamed, S. Dimopoulos and G. Dvali, Phys. Lett. B 436, 257 (1998).

[2] L. Randall and R. Sundrum, Phys. Rev. Lett. 83, 4690 (1999); Phys. Rev. Lett. 83, 3370 (1999).

[3] M. Cavaglia, Int. J. Mod. Phys. A 18, 1843 (2003); P. Kanti, Int. J. Mod. Phys. A 19, 4899 (2004).

[4] S.W. Hawking, Nature 248, 30 (1974); Comm. Math. Phys. 43, 199 (1975).

[5] S. Dimopoulos and G. Landsberg, Phys. Rev. Lett. 87, 161602 (2001).

[6] C. M. Harris, P. Richardson and B.R. Webber, JHEP 0308, 033 (2003).

[7] S. Dimopoulos and G. Landsberg, Black hole production at future colliders, in Proc. of the APS/DPF/DPB Summer Study on the Future of Particle Physics (Snowmass 2001), edited by N. Graf, eConf C010630, P321 (2001).

[8] E.J. Ahn and M. Cavaglia, Phys. Rev. D 73, 042002 (2006).

[9] M. Cavaglia, R. Godang, L. Cremaldi and D. Summers, Comput. Phys. Commun. 177 (2007) 506.

[10] G.L. Alberghi, R. Casadio, A. Tronconi, J. Phys. G G34 (2007) 767; G.L. Alberghi, R. Casadio, D. Galli, D. Gregori, A. Tronconi and V. Vagnoni, "Probing quantum gravity effects in black holes at LHC," hep-ph/0601243.

[11] D.-C. Dai, G. Starkman, D. Stojkovic, C. Issever, E. Rizvi, J. Tseng, Phys. Rev. D77 (2008) 076007.

[12] J.A. Frost, J.R. Gaunt, M.O.P. Sampaio, M. Casals, S.R. Dolan, M.A. Parker, B.R. Webber, JHEP 0910 (2009) 014; M.O.P. Sampaio, "Production and evaporation of higher dimensional black holes," Ph.D. thesis, http://www.dspace.cam.ac.uk/handle/1810/226741.

[13] CMS Collaboration, V. Khachatryan et al., Phys. Lett. B 697 (2011) 434.

[14] S. C. Park, Phys. Lett. B 701, 587 (2011).

[15] G. Landsberg, J. Phys. G 32, R337 (2006).

[16] C.M. Harris, M.J. Palmer, M.A. Parker, P. Richardson, A. Sabetfakhri and B. R. Webber, JHEP 0505, 053 (2005).

[17] A. Casanova and E. Spallucci, Class. Quant. Grav. 23, R45 (2006). 
[18] R. Casadio, B. Harms and Y. Leblanc, Phys. Rev. D 57, 1309 (1998); R. Casadio and B. Harms, Phys. Rev. D 58, 044014 (1998) and Mod. Phys. Lett. A14, 1089 (1999).

[19] R. Casadio, B. Harms, Entropy 13 (2011) 502-517.

[20] B. Koch, M. Bleicher and S. Hossenfelder, JHEP 0510, 053 (2005).

[21] S. Hossenfelder, Nucl. Phys. A 774, 865 (2006).

[22] D.M. Gingrich, JHEP 1005 (2010) 022.

[23] T. Sjostrand, S. Mrenna and P. Skands, JHEP 0605, 026 (2006).

[24] K.S. Thorne, in Magic without magic, edited by J. Klauder (Freeman, 1972).

[25] G. L. Alberghi, R. Casadio, O. Micu, A. Orlandi, JHEP 1109 , 023 (2011).

[26] D. Ida, K. -y. Oda and S. C. Park, Phys. Rev. D 67, 064025 (2003) [Erratum-ibid. D 69 (2004) 049901]; Phys. Rev. D 71, 124039 (2005); Phys. Rev. D 73, 124022 (2006).

[27] S. Creek, O. Efthimiou, P. Kanti and K. Tamvakis, Phys. Rev. D 75, 084043 (2007); Phys. Rev. D 76, 104013 (2007); G. Duffy, C. Harris, P. Kanti and E. Winstanley, JHEP 0509, 049 (2005); M. Casals, P. Kanti and E. Winstanley, JHEP 0602, 051 (2006); M. Casals, S. R. Dolan, P. Kanti and E. Winstanley, JHEP 0703, 019 (2007);

[28] C. Herdeiro, M.O.P. Sampaio and C. Rebelo, JHEP 1107 (2011) 121.

[29] C. Herdeiro, M.O.P. Sampaio and M. Wang, Phys. Rev. D 85 (2012) 024005; M.O.P. Sampaio, JHEP 1002 (2010) 042; JHEP 0910 (2009) 008.

[30] P. Nason, JHEP 11, 040 (2006); S. Frixione, P. Nason and C. Oleari, JHEP 11, 070 (2007). S. Frixione, P. Nason, and G. Ridolfi, JHEP 09, 126 (2007). 

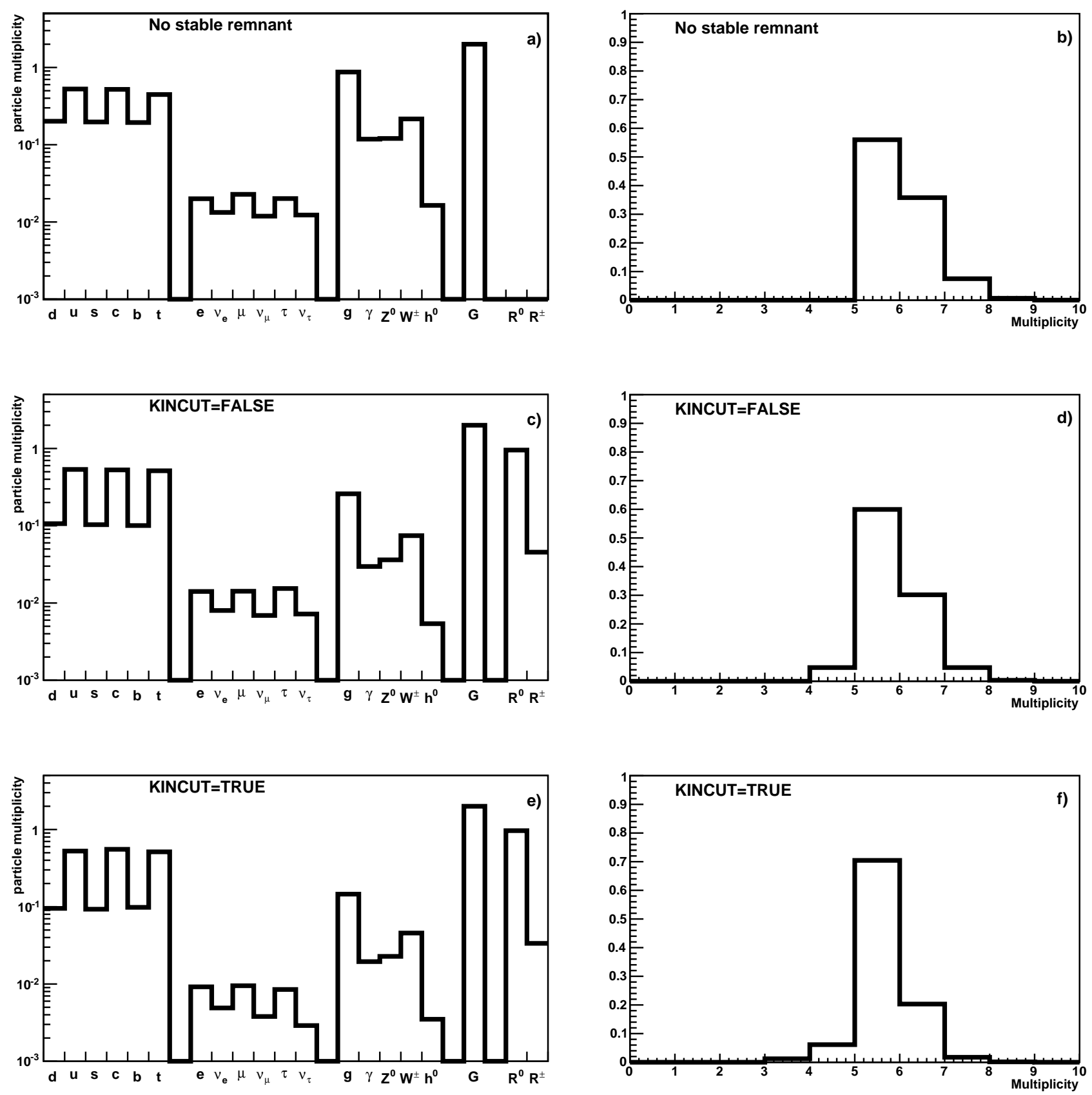

Figure 2: Primary decay products of the $\mathrm{BH}$ (panels a, c, e) and multiplicity (panels b, d, f) for no remnant, KINCUT=FALSE and KINCUT=TRUE, respectively. The plots have been obtained using Monte Carlo samples generated at $7 \mathrm{TeV}$, with $M_{\mathrm{G}}=2 \mathrm{TeV}$ in $D=8$. 

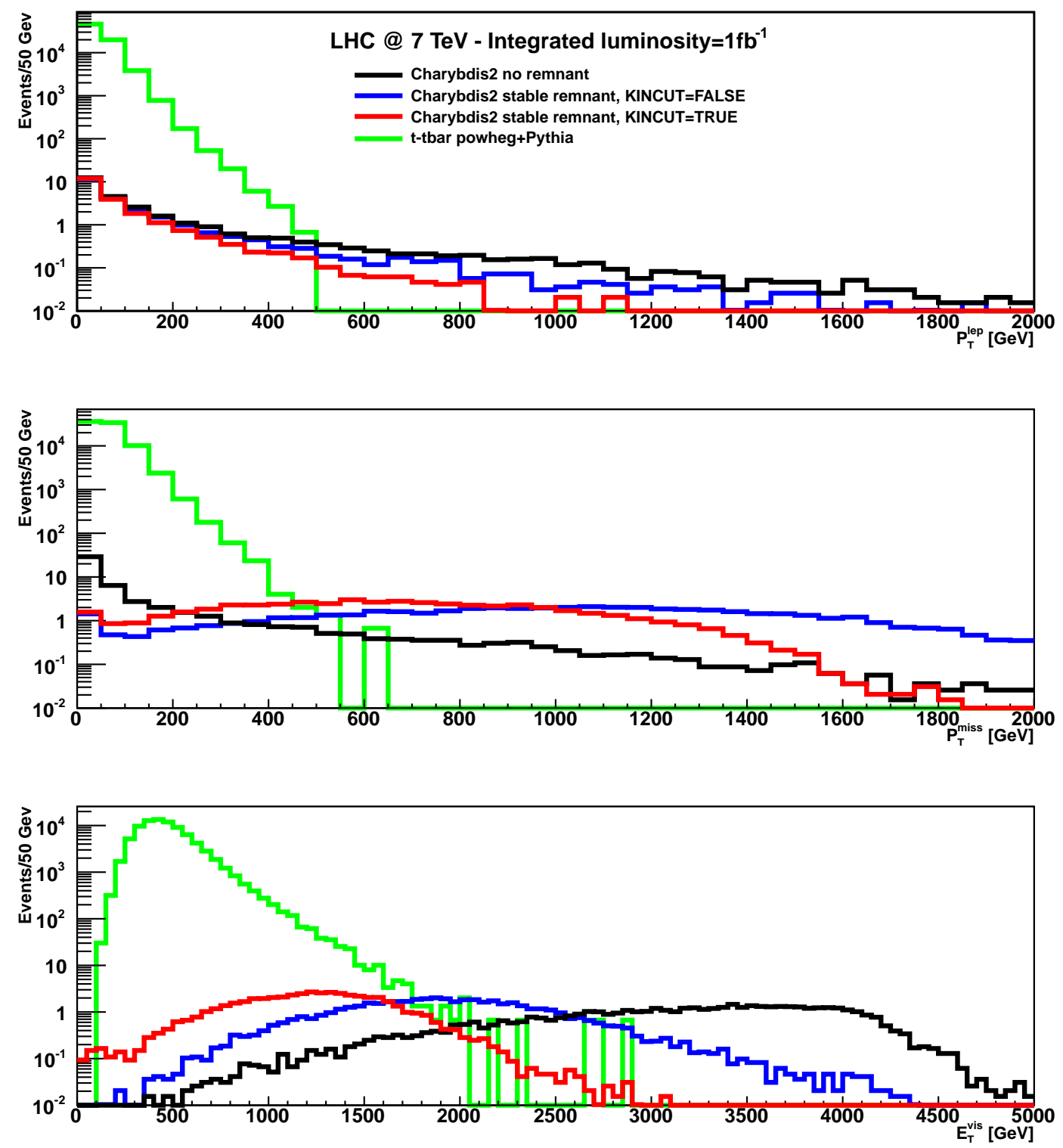

Figure 3: Distributions of $P_{T}^{\text {lep }}, P_{T}^{\text {miss }}$ and $E_{T}^{\text {vis }}$ for BH events with different remnant scenarios and for the $t \bar{t}$ background. The $\mathrm{BH}$ distributions have been obtained using the simulated samples at $\sqrt{s}=7 \mathrm{TeV}$ with $M_{\mathrm{G}}=2 \mathrm{TeV}$ in $D=8$. 

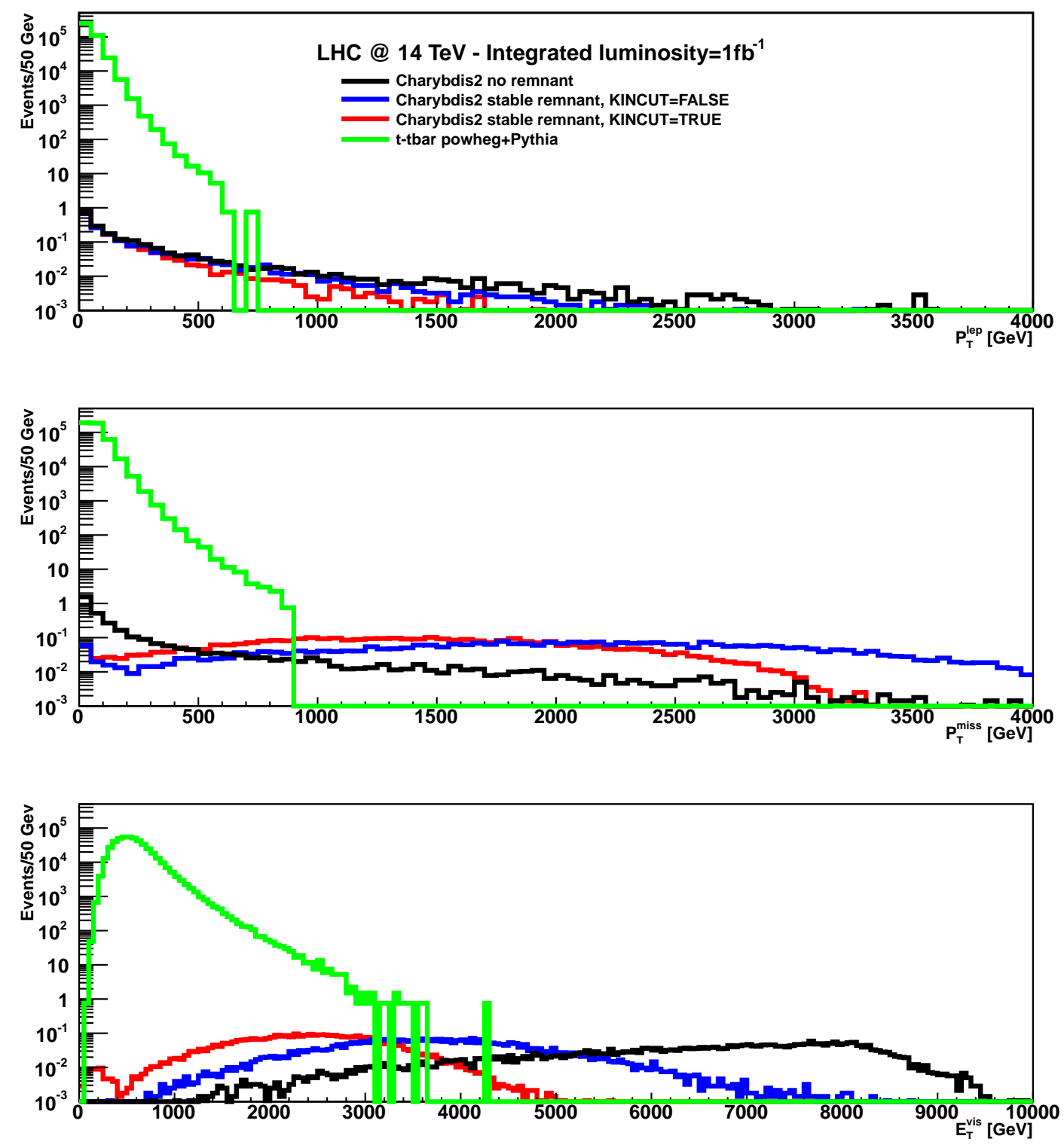

Figure 4: Distributions of $P_{T}^{\text {lep }}, P_{T}^{\text {miss }}$ and $E_{T}^{\text {vis }}$ for BH events with different remnant scenarios and for the $t \bar{t}$ background. The $\mathrm{BH}$ distributions have been obtained using the simulated samples at $\sqrt{s}=14 \mathrm{TeV}$ with $M_{\mathrm{G}}=4 \mathrm{TeV}$ in $D=6$. 

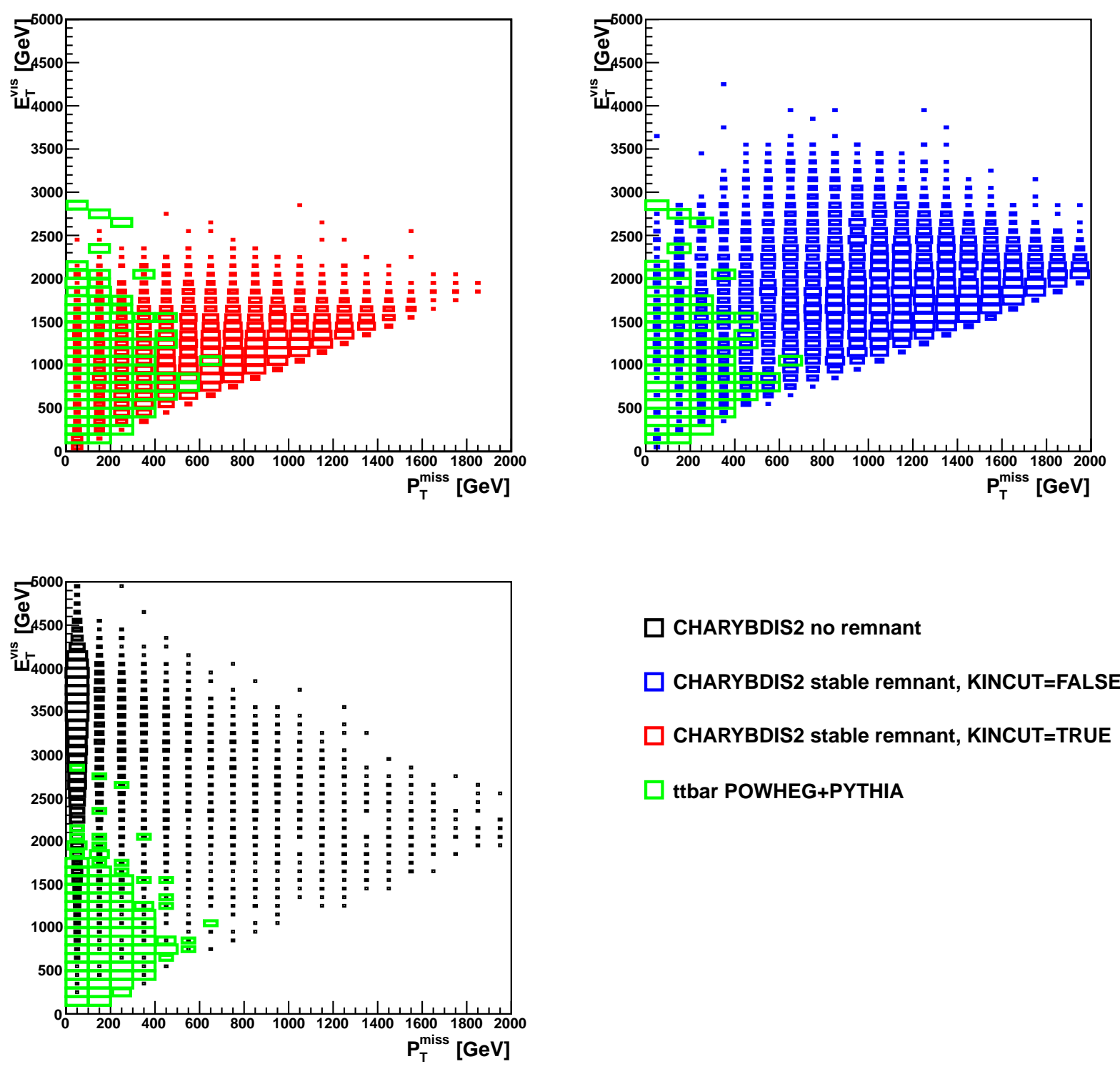

$\square$ CHARYBDIS2 no remnant

$\square$ CHARYBDIS2 stable remnant, KINCUT=FALSE

CHARYBDIS2 stable remnant, KINCUT=TRUE

ttbar POWHEG+PYTHIA

Figure 5: Distributions of signal and background events in the $E_{T}^{\mathrm{vis}}-P_{T}^{\mathrm{miss}}$ plane for the different remnant scenarios. The $\mathrm{BH}$ distributions have been obtained using the simulated samples at $\sqrt{s}=7 \mathrm{TeV}$ with $M_{\mathrm{G}}=2 \mathrm{TeV}$ in $D=8$. 